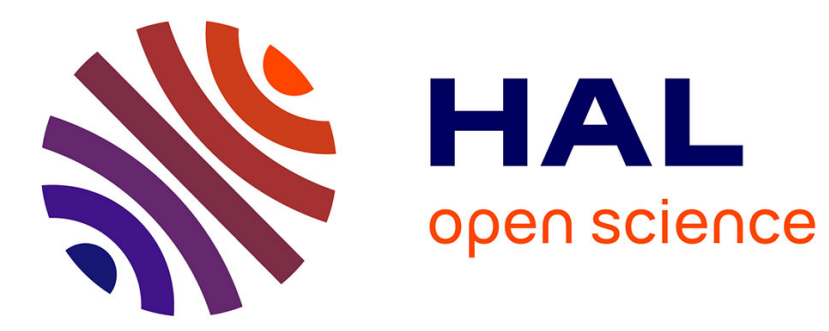

\title{
Le cirque Molier et ses athlètes aristocrates à la Belle Époque: succès mondain et controverse politique
}

\author{
Gabrielle Houbre
}

\section{To cite this version:}

Gabrielle Houbre. Le cirque Molier et ses athlètes aristocrates à la Belle Époque: succès mondain et controverse politique. Modern and Contemporary France, 2007, 15 (4), pp.497-511. 10.1080/09639480701627626 . hal-02455116

\section{HAL Id: hal-02455116 \\ https://hal.science/hal-02455116}

Submitted on 28 Jan 2020

HAL is a multi-disciplinary open access archive for the deposit and dissemination of scientific research documents, whether they are published or not. The documents may come from teaching and research institutions in France or abroad, or from public or private research centers.
L'archive ouverte pluridisciplinaire HAL, est destinée au dépôt et à la diffusion de documents scientifiques de niveau recherche, publiés ou non, émanant des établissements d'enseignement et de recherche français ou étrangers, des laboratoires publics ou privés. 
Gabrielle HOUBRE, «Le cirque Molier et ses athlètes aristocrates à la Belle Époque : succès mondain et controverse politique », Modern and Contemporary France, vol. 15, Number 4, November 2007, pages 497-511.

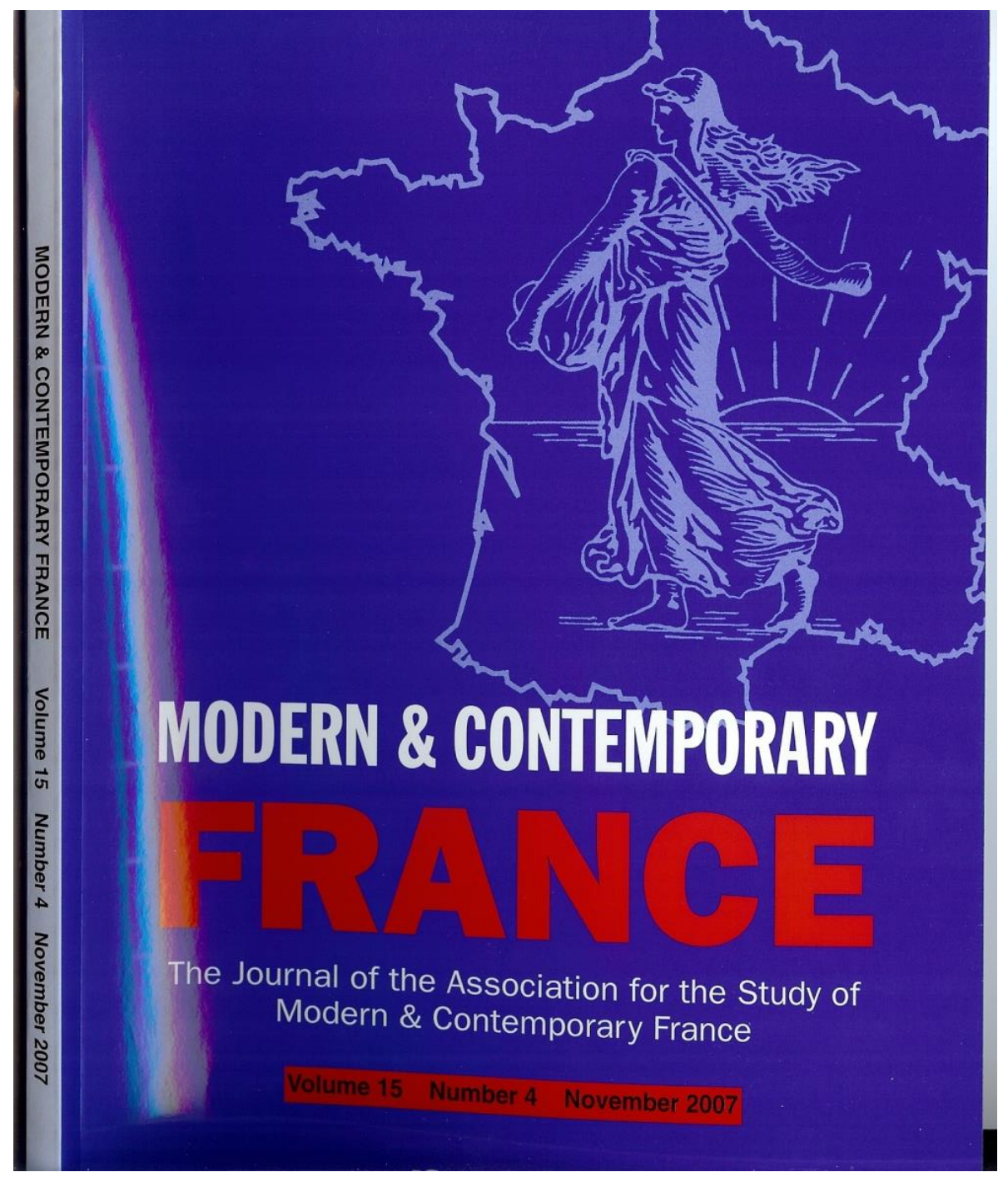




\title{
Le Cirque Molier et ses athlètes aristocrates à la Belle Époque: succès mondain et controverse politique
}

\author{
Gabrielle Houbre
}

Au carrefour de l'histoire sociale et culturelle et du genre (gender), cet article propose une réflexion sur une pratique privée qui déborde, au cours d'une vive polémique entretenue par la presse nationale, dans l'espace public. En 1880, Ernest Molier fonde un cirque privé exceptionnel, les deux représentations annuelles de ce cirque l'impose pourtant comme un must de la saison parisienne jusqu'à la mort de Molier en 1933: La chronique mondaine puis les rubriques politiques s'emparent bientôt de l'évènement pour s'émouvoir de ce que les deux représentations s'adressent alternativement aux femmes $d u$ monde puis du demimonde. Mais c'est surtout la participation active des héritiers de grandes familles aristocrates au spectacle qui est âprement discutée, certains journaux la stigmatisant comme un signe de décadence morale et politique à une époque où les débats sur la dégénérescence de la race battent leur plein.

Le 21 mars 1880 se déroule à Paris la première représentation offerte par un cirque hors du commun. Son fondateur, Ernest Molier, est un bourgeois assez riche pour s'offrir le luxe d'une piste de cirque érigée au cour de sa propriété, rue Bénouville, près du Bois de Boulogne, assez bien inséré dans les cercles mondains pour avoir pu recruter une troupe d'amateurs parmi ses amis aristocrates et grands bourgeoisauxquels viendront s'ajouter quelques artistes féminines - et assez audacieux pour présenter un véritable spectacle alternant numéros équestres avec numéros de force, d'adresse et de divertissement. Le public rassemble plusieurs centaines d'invités distingués dans le beau monde pour les hommes, et dans le demi s'agissant des femmes avant que, dans un second temps, aristocrates et grandes bourgeoises ne réclament d'assister à l'événement (Molier 1905, chap. 1). Enhardi par la réussite de cette séance encore inédite de sociabilité high life, Molier répète trois mois plus tard son spectacle

Correspondence to: Université Paris Diderot-Paris 7 / Institut Universitaire de France, 147 rue de Bercy, 75012 Paris, France. Email: houbre.gabrielle@univ-paris-diderot.fr 
498 G. Houbre avant de l'ancrer durablement dans le temps, puisqu'il réussit l'exploit de faire donner par sa troupe deux à trois

jusqu’à sa mort, en 1933.

Les soirées du cirque Molier, rares et sélectives, donc doublement précieuses, Les rendez-vous les plus courus de la vie s'imposent immediatement comme Puir certains des parisienne: le Tout-Paris s'arrache les invitations qui lui permet rivaliser avec celles de siens se mettre en scène dans des attractions susceptibles dent la rue Bénouville de professionnels et, bien vite, les plus brillants équipages tirent la rue Benouville de l'obscurité dans laquelle elle végétait jusqu'alors (Le Figaro, 6 juillet 1880). Le succes robscurite dans laquelle ellant dans son sillage les critiques et bientôt la presse consacre grandissant draine cependant dans 2 janvier 1885, le très catholique et très conservateur l'événement en s'en emparant. Le 2 janvier 1885 , les maitresses et des épouses dans les L'Univers, après avoir condamné l'alternance des maitresses aristocrates s'exhibant en deux publics féminins, s'offusque de l'inconvenance de ces aristocrates s'exh cette fin de siècle maillots d'athlètes quand ce n'est pas en costumes de clowns. Dans cette fin de siecte mallots dea vivent comme crépusculaire, ${ }^{1}$ le cirque Molier échappe à sa vocation que beaucoup vivent comme crepusculvé pour devenir un catalyseur des idées, des initiale de simple divertissement prive pour devenir des notions de race, d'hérédité angoisses ou des fantasmes qui agitent les esprits autour des notions deblesse et même des et de dégénérescence mais aussi du rỏle social et politique de la nte, est un authentique rapports régissant les deux sexes. Le cheval, dans ce contexte, est un authentique rapports régissant les de passion que lui révélateur des termes et des enjeú d'animal est l'attraction porte le cavalier, dresseur et pédagogue, Ernest Molier. Luar duquel s'articule le principale du cirque en même temps que le pivot aulesse le délaissent pour lui programme. Et c'est lorsque les représentants de la noblesse le delaissent pou pied la

préférer des numéros de gymnastique, de force ou

"Celui-là est un véritable Centaure " (Vaux 1888a, p. 109)

(lenfance et la jeunesse d'Ernest Molier, sinon le peu On ne connait presque rien sur lenfance et la jeunesse de Er son cirque (Molier 1905, qu'il a lui-même raconté en avant-propos de son ouvrage sur son cirque (Molier la bourgeisie pp. I-III). Né en 1844 dans la Sarthe, ${ }^{2}$ il est issu d'une bonne famille de la bourgeoise-t-il, qui compte des membres dans la magistrature et dans larmée et trésorier-payeur au des nobles et des « décorés». Son père, Jean Adrien Molier, est trésorier-payeur a des ne Mans, passion precoce que son fls a nourrie ponte, le jeune homme s'installe à Paris dès $\mathrm{s}$ études apparemment brèves et peu brillantes, le jeune homme loisirs que lui autorise une majorité. Il profite alors essentiellement à cheval des loisirs que lacer une activite fortune assez importante pour qu'il n'ait jamais eu besoin dexercer une activiteur $3^{3}$ salariée et décide de « devenir écuyer, sinon professionnel, du moins amateur ". les 1t expentiellement en autodidacte une science equestre linresque, Ies Il expériment envoye promener » selon sa gens du métier, « jaloux de leur art myst'extérieur, il prétend dédaigner la Haute École propre expression. Cavalier avant tout d' extérieur, il s'intéresse à l'équitation savante de classique qui, dit-il, « ratatine » les chevaux, mais il sinteresse à Pequitation sand

\section{G. Houbre}

à apprendre la monte à califourchon à ses apprenties, " convaincu que lorsqu'une femme sait monter en homme, elle contracte une solidité et un maniement du cheval qu'elle n'aurait jamais pu acquérir en amazone » (Molier 1905, p. 12).

Hormis quelques chevauchées nocturnes, l'écuyer ne se risqua pourtant pas à sortir de l'ombre de son manège ses apprenties écuyères, "ne voulant pas les rendre ridicules et les exposer par leur posture grotesque à la risée des promeneurs " (Molier 1911, p. 280 et sa lettre dans André 1908, p. 198). S'il lance avec bonheur la mode des écuyères travesties en homme-notamment en uniforme du Cadre noir de Saumuril respecte les usages du monde qui, dans les années 1880 , bannissent encore la monte à califourchon pour les femmes. Aussi, on ne saurait s'égarer à penser qu'il ait voulu participer en quoi que ce soit à l'élan féministe qui prenait de la vigueur dans les années 1880 (Klejman \& Rochefort 1989, p. 356). Plus tard, il se flattera au contraire d'être " un fervent admirateur de la grâce féminine ", affirmant ne trouver rien de plus " laid " ni de plus " disgracieux " qu'une femme à califourchon, rejoignant par là le sentiment vulgaire de ses pairs (lettre de Molier dans André 1908, p. 198 et dans Pour bien faire du sport 1912, p. 210). Et pour qu'aucun doute ne puisse subsister, il ajoute:

Je ne puis supporter la femme qui se donne des allures masculines. Je dois dire même que, pour moi, chaque fois qu'elle se fait homme d'une manière quelconque, je la renie complètement, et la grande attraction que j'ai pour son sexe se transforme en

répulsion. Ainsi, pas plus de femmes à califourchon que de femmes députés, notaires, avoués, cochères, etc. (Lettre de Molier dans André 1908, p. 198)

L'ambivalence de Molier, dont la politique sociale hésite entre public et privé, entre respect avisé des convenances et réalisation volontiers transgressive de ses désirs particuliers, est l'un de ses traits distinctifs. Elle se lit dans tout l'ordonnancement de son cirque, du choix des compagnons appelés à fouler la piste avec lui jusqu'à l'élection des happy few invités à les applaudir, en passant par la distribution des numéros.

\section{L'Économie sélective du cirque Molier ou les raisons d'un succès mondain}

Comme le relève avec justesse Robert Milton dans Le Figaro du 6 juillet 1880, « Molie est purement un amateur riche qui s'offre le luxe d'avoir un cirque chez lui, comme d'autres propriétaires ont une salle de théâtre dans leur hôtel ». De fait Molier a suffisamment voulu son cirque pour déménager de son hôtel de la rue Blanche-trop exigu—et venir s'installer près du stratégique Bois de Boulogne, où il comptait bien monter chaque jour, et faire ériger à grands frais une piste réglementaire de treize mètres de diamètre. Au premier bâtiment d'allure incertaine, construit en planches et en plâtras, couvert en tuiles et comprenant un étage dévolu à la loge des artistes, succédera en 1898 un véritable cirque en bois, plus spacieux, occupant près de la moitié de la surface bâtie de la propriété. ${ }^{9}$ L'entreprise était privée et à but non lucratif (troupe amateur et public non payant); environ $20000 \mathrm{f}$. de frais annuels d'entretie s'ajoutaient aux capitaux initiaux de construction: ${ }^{10}$ il ne faut donc pas s'étonner de ce que la presse revienne plusieurs fois sur le « luxe princier » déployé par ce bourgeois rentier. cirque à laquelle l'initie Alfred Bradbury, le seul professeur qu'il se reconnaisse. Henry lui adjoint Victor Franconi et Jame Thétard, dans sa Merveilleuse histoire du cirque, lui adjoint un écuyer complet, sachan Thétar é déclare qu'« à vingt-cinq ans, M. Molier était un écien qu'exécuter un pas Fillis et déch e clus difficile, aussi bien que exécuter le cheval le p. 235). mettre au passage au panneau ou cheval nu " (Thetard 1978, p. 235 ). de deux des plus classiques sur panneau sa "disponibilité ", ${ }^{4}$ Molier put et sut Réservant à la pratique de lequitation sáthodique: «Après bien des annees d'un s'astreindre à une discipline patiente et méthodicionné, je parvins à connaitre assez s'astrail qui eût été pénible pour un autre moins passionné, je pates sortes, et former des travall que dresser des chevaux, des animaux de toutes sor moi-même à ces mon affaire pour-je très fier, précise-t-il, d'être arrive seul et par moi-méne à ces élèves. Aussi suis je tis fó amateur, il n'envisageait la pratique de l'art equestre que résultats ». Bien que denmes reprendre ses termes, et ne souffrait en la comme un " travail ", un " metier ", pour Vaux, qui contribua dans ses ouvrages matière d'aucun dilettantisme. Le baron de Vaux, quí contribua dans ses aptitude maticre d'teuse réputation d'homme de cheval de Molier, insiste sur ses apt, Molier asseoir la flat comme Paul Haynon qui note qu'en quarante-cinq ans, Mot même de dresseur, tout con dressé plus de quarante chevaux, ma la célèbre écuyère Blanche Allarty. Il semble des chameaux, montés en haute ecole par la celcerniers la méthode Baucher et que, d'ailleurs qu'il ait appliqué au dressage de ces derniers la méth - en vain-son procéd

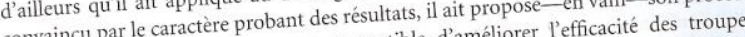
convaincu par le caracterre comme susceptible d'améliorer l'efficacié des troupes au ministère de la guerre commés méharistes à l'œuvre dans la colonisation aficaine jeunes gens appartenant à la jeunesse Sportsman accompli comme beaucoup des jeclaireurs où Jacob enseignait l'épée, et élève de dorée, escrimeur assidu au Cercle des éclaireurs oú acobval, comme le prouvent les très doree, escrist Lecour en boxe, de presse qui lui sont consacrés de son vivant et lors dé en selle son nombreux articles de pre $1^{\text {er }}$ août 1933, à quatre-vingt-neuf ans, Gaulois 1884; Ami du peuple du soir 1933), dernier spectacle: "Centaure " (Le Gaulois 1884, Amaute École " (Le Clairon 1884) dernier spectinfatigable » (Le Gaulois 1884), " roi de la Haute Ecole " (Le Clge), tels sont les " horseman infatigable "Le " écuyer hors ligne " (L'Indépendance belge), tels de cheval « dresseur incomparable qualificatifs les plus explicites qui peuple du soir 1933; Le Temps 1933; Action française passionné (Le Figaro 1933; Ami du peuple du soir corde à son arc, celle de la pédagogie. 1933). Pour autant, Molier a ajouté une autre corde à son aresque exclusivement recrutée Très vite il a en effet souhaité former des élèves qu'il a presque exclup d'entre elles sont Très vite il a en effet filles qui se destinaient au cirque. Beaucoup Lankast, Pâquerette, parmi les jeunes filles qui sées comme Fanny Lehmann, Louisa Lankast, Páquerete devenues des écuyeres réputées cormm, et surtout celle qui s'est imposee comme la plus Irma Viollat, Camille Van Walberg, et surtout celly, que Z lança en 1890 et qu il epousa grande écuyère de la Belle Epoque, Blanche Allarty qúminine s'est beaucoup développee par la suite. ${ }^{8}$ À une époque où, certes, l'équitation feminine (monte en amazone, cheval par la suite. A unatique selon une codification spécifique (monte en la cavalière) visan mais ou elle se pratique se our la monture, toilette d'amazone pour la cavaliére couple de dame, selle à fourches pour mesure nécessaires qui doivent animer la coubre 1998 à souligner la grâce et la mesure nécessent que dans sa gestuelle (Houbre 1998 : pp. $85-99)$
Rue Bénouville, Molier, tour à tour cavalier, dresseur et instructeur, s'abandonne quotidiennement à sa passion de l'équitation. Il reçoit en outre la visite régulière de ses compagnons sportsmen, aristocrates et bourgeois fortunés, tous cavaliers, escrimeur ou boxeurs, qui viennent profiter de son manège, à une époque où les activités sportives suscitent un réel engouement de la part des membres de la haute société parisienne: ${ }^{11}$

Ils apportèrent chacun leur bagage sportif. Pendant que je dressai mes chevaux, les escrimeurs se mirent à ferrailler au milieu de la piste, les forts en biceps levèrent des

poids, et les amateurs de gymnastique firent leur Léotard au-dessus de ma tête.

Si bien qu'un beau jour, je ne sais lequel de nous m'engagea à organiser une petite

soirée équestre. ${ }^{12}$ (Molier 1905, p. 5 )

Car, pour Molier et bien qu'il soit conscient du déclin du cirque équestre concurrencé même moment par le music-hall, l'étoile du spectacle doit demeurer le cheval; et de fait, jusqu'à la dernière représentation en 1933, celui-ci est le prétexte à la majorité des numéros, que ces derniers relèvent de la haute école, du dressage en liberté, de la pantomime, de la voltige, ou d'excentricités diverses. Molier, lui-même, se réserve le premier rôle: il ouvre le spectacle en haute école et montre ensuite son habileté d'écuyer de cirque en exécutant des pas de deux sur panneau ou cheval nu, ou en présentant des chevaux en liberté. ${ }^{13} D^{\prime}$ 'autres numéros hippiques, de divertissement ou d'équitation académique, sont offerts par ses amis, tous nobles ou militaires, ce dont l'on ne s'étonne pas tant le cheval est l'élément nodal d'une identité masculine par essence aristocratique et guerrière (Houbre 2003, p. 15).14 À l'exception d'un comte, qui s'expose au trapèze et à la barre fixe, et d'un autre, qui joue au clown, les autres participants, bourgeois plus ou moins aisés et plutôt plus que moins, se partagent des partión de force, d'adresse et de divertissement oscillant entre démonstrations sportives et exercices de cirque. ${ }^{15}$

Si Molier n'a eu aucune peine à composer sa troupe dans sa partie masculine, il se trouve fort embarrassé au moment de la compléter par l'« élément féminin ", « jugé indispensable "; cette " terrible question ", selon son expression, l'incite à s'inquiéter du monde où chercher ses collaboratrices. Son premier réflexe, dans un souci de cohérence, est de songer au même que celui des messieurs, d'autant que, dans ces années 1880, les aristocrates, plus libres de s'intéresser aux activités sportives que les bourgeoises, se doublent de plus en plus souvent de sportswomen accomplies (Vaux Les Femmes de sport 1885 et Saint-Martin 1989, pp. 28-30). Cependant, malgré la candidature de plusieurs amazones parmi les plus hardies, ${ }^{16}$ Molier renonce aux femmes du monde dont la présence aurait immanquablement entraîné l'exclusion des autres, c'est-à-dire des cocottes, Or, Molier était alors encore garçon et à ce titre avouait en connaitre «pas mal au théâtre, au concert, au Bois et même en dehors de ces trois promenoirs favoris du monde galant "; il était même bon garçon: ne se sentant pas le courage de leur fermer sa porte le soir de la fête, il préféra se passer des « légitimes » de ses amis, sans compter qu'il redoutait que ces maris jaloux des prérogatives que leur conféraient le droit et l'usage, « empêch[ent] leurs femmes de cascader devant eux à pied et à cheval " (Molier 1905, pp. 7, 8). Il fit donc appel à quelques artistes 
professionnelles, en particulier les écuyères Fanny Lehmann, Emma Bradbury, Renée Dudley et l'actrice Alice Lavigne. Elles vinrent renforcer la troupe qu'il formait avec ses compagnons, tous authentiques amateurs bénéficiant néanmoins d'assez de loisirs pour sonossionnel (Thétard 1978, p. 235).

"Amphitryon aimable et magnifique ", comme le proclame Paris-Journal dans son numéro du 21 juin 1881, Molier trie sur le volet ses trois à quatre cents invités qu’i régale, outre le spectacle, d'un dîner sans façons: ${ }^{17}$

Tout ce que Paris compte de sportsmen distingués, tous les habitués du Bois le matin, s'étaient donné rendez-vous hier soir chez M. Molier qui offrait à ses amis une fête dont on se souviendra longtemps dans le high life parisien. Nous trouvions l'élite de la magistrature, de la médecine, de la finance et de la noblesse. ${ }^{18}$

La sélection sociale est posée comme règle de base et Molier n’y déroge pas jusqu’à sa mort, même si, au fil des années, elle se démocratise quelque peu:

Ici, spectateurs et acteurs sont des gens de même éducation, de même milieu, qui se onnaissent tous. (...) Ils entendent continuer à se divertir librement, entre gens du monde, et laisser dehors qui leur déplaît (...) La porte est close pour les gens de théâtre. (Le Roux 1889, p. 242)

Là, dans cet entre-soi élargi à d'aimables demi-mondaines et demoiselles du spectacle, réside sans doute une des clefs du succès retentissant de l'entreprise qui dépasse largement les espérances de ses promoteurs. Bientôt quelques articles s'enthousiaslargement les esperanes de ses proluent une « idée vraiment neuve et sûrement piquante" (Le Figaro 1880). Les comptes-rendus abondent dans les chroniques mondaines, encensant les performances équestres et athlétiques proposées par ces moteurs stylés et égrenant les figures vedettes du Tout-Paris présentes dans le public telles que le Prince Sagan ou le duc de Morny.

Il est intéressant de constater qu'à partir du moment où la représentation du cirque Molier s'imposait comme un événement mondain, les épouses se rebellèrent contre leur éviction et se refusèrent, au moins comme spectatrices, à laisser le champ libre aux mairteses de leurs maris. Cédant à leurs instances pressantes, Molier organise, à parti de 1882, une double représentation du même spectacle pour ses deux public féminins, habitude qui perdura jusqu'à la fin du cirque:

L'élément féminin, qui assiste à ces réunions très recherchées, se divise, on le sait, en deux catégories absolument tranchées. Les parentes de ces messieurs, qui, quand elles mettent le pied dans l'établissement de M. Molier, savent qu'elles rentrent dans un salon aussi rigoureusement fermé que n’importe quel logis aristocratique. Quant à leurs amies, elles ont leur jour absolument distinct, et les premières comme les dernières sont convaincues qu'aucune lettre d'invitation n'a pu s'égarer au contrôle. Ces deux camps vivent d'ailleurs en fort bonne intelligence,

Cette disposition, qui reflétait la bipolarisation traditionnelle des amours mette en respectait l'aménagement istinct (Houbre 1996, pp. 311-337), fit néanmoins beaucoup jaser en societe et dans (a) 20 dauber ces petits arrangements avec la 列 morale, les feuilles modérées épinglant l'alternance des firaro 1883 et 28 mai 1886), qui en sont moins " voire qui "n'en sont pas " (Le Figaro 1883 et deux fournées les autres-de droite comme de gauche-se dechainant sur " les dentales de grande comprenant les « marquises authentiques " d'une part, les " horizontales de grande marque " de l'autre (L'Écho de Paris, 1885 ou la variante " horizontales de la haute noce " du Radical, 1886), ou encore « l'aristocratie galante " ou " dessus du panier a quinze sous » opposée aux « femm (Le Voltaire 1885). que des pêches à trente sous " (Le Voltaire 1885). La boutade bien élevée non plus que le ricanement graveleux n'entamerent en rien l'engouement des élites pour leur cirque, lesquelles en firent rapidement l'un des moments-phares de la saison parisienne et, par là-meme, le publicisel en le mettant en de théatre et des romans le consacrèrent comme réferent culturel en le mettant cette scène. ${ }^{22}$ La presse donna davantage d'ampleur encore au phénomène en discutant cette scèn innovation qui "bouleversait tous les spectacle devant un public restreint, composé gentleman, tout en se donnant en spectacle devant un pubse leurs pairs" ? Pendan d'hommes du meilleur monde, venant juger des prouessalifie de « révolution » (1888b, quelques années, le débat sur ce que le baron de Vaux qualife de des analyses politiques p. 112), quitta les rubriques mondaines policées pour alimenter des analyses politiques p. 112), quitta les rubriques mondrant, en même temps que la cohésion sociale et et sociales contradictoires montrant, en l'aristocratie et de la bourgeoisie, la force de culturelle des couches les plus élevées de l'aristocratie et de la $\mathrm{III}^{\mathrm{c}}$ République (Mayer l'attraction que ce 1983, p. 107)

\section{e Corps exhibé de la noblesse ou les raisons de la controverse}

L'essentiel du débat se situe en 1885-1886, avant le déclenchement de la crise boulangiste, dans un clerry installé depuis février 1883, voit dépression économique. Le second cabinet Ferry, installé depuis fin par tomber le certes la plupart des grandes lois républicaines votees, mais il fint par tomapartistes, 30 mars 1885. La droite, qui s'était déchirée aux élections de 1881 entre bonapartistes légitimistes et orléanistes, se remobilise fortement pour celles d'octobre 1885 et fait légitimistes et orleanistes, sue doubler son nombre d'élus à la Chambre des députés. ${ }^{23} \mathrm{La}$ presse, qui jouit depuis la loi du 29 juillet 1881 d'un régime très libéral répliquant aux contrainion. imposées par l'ordre moral, est en plein essor, particulierement les feuilles d'opinion. Dans ce contexte effervescent, L'Univers, qui demeure après la mort de Louis Veuillot ${ }^{24}$ un journal de combat ultramontain et ultra conservateur, lance une pierre, le 2 janvier 1885, dans le "cirque d'amateurs " et, par ricochets, dans les d'articles rédactions de plusieurs de ses confrères. Il reprend en effet des extré des temps oi favorables à la troupe de Molier qui, selon lui, " Connent une thiste idee des favora d'une " décadence morale " digne de l'antique [ils] vivent ", c'est-à-dire ceux d'une " decadence mor cirque soit devenu une « institution parisienne "
504 G. Houbre

et qu'il soit " entré dans les mœurs comme la Fête nationale ou la séance annuelle de l'Académie ", s'indigne du pragmatisme guère moral qui fait se succéder deux publics féminins, épouses et maitresses et, surtout, se scandalise du fait que des hommes du meilleur monde puissent s'ingénier à « lutter avec les vrais écuyers et avec les clowns de profession ». L'Univers conclut sa recension par ces quelques lignes affligées:

Nous ne ferons pas d'autres réflexions; elles n'ajouteraient rien à la pénible impression que doit produire le simple exposé des faits. Mais ce qu'il faut dire trè haut, c'est que la France serait bien perdue si elle ne devait compter que sur cett étrange sorte de régénérateurs. (L'Univers 1885)

Avec les réactions très politiques de journaux de tous bords, la polémique-latente depuis 1882 et l'affichage de la double morale sexuelle dans le choix du public féminin-éclate pour rebondir l'année suivante, lorsque Molier et sa troupe acceptent de donner une représentation exceptionnelle de leur spectacle au profit de l'CEuvre de l'Hospitalité du Travail patronnée par la duchesse d'Uzès, personnalité assez peu conformiste pour avoir été, notamment, la première femme maitre d'équipage de chasse à courre. ${ }^{26}$ Beaucoup, $\mathrm{y}$ compris parmi les plus modérés, ${ }^{27}$ s'offusquent alors de voir, au nom du devoir de charité, la fine fleur de l'aristocratie et des salons parisiens se divertir dans les loges et les gradins ou s'ébattre sur la piste du confortable et luxueux Nouveau cirque, celui de la rue Bénouville étant trop petit pour l'occasion. ${ }^{28}$ Le déba se focalise alors sur l'éventuel discrédit d'une noblesse qui prétend jouer aux artistes de cirque, sur la pertinence de l'exhibition des corps et de l'utilisation de la force et, plus généralement, sur le degré de sa dégénérescence et de sa capacité à assumer son rôle parmi les classes dirigeantes.

Car ici les représentants de la bourgeoisie importent peu. Le Radical, dans son numéro du 25 mai 1885, dénombre bien quelques roturiers enrichis membres du Jockey Club dont Le Voltaire du 29 mai 1886 prétend qu'on "chuchote tout bas » les noms, mais ils sont éclipsés par la présence des gentilshommes et non des moindres. Le Rappel, dans un article du 10 janvier 1885 persiflant L'Univers qui " grogne et ronchonne ", a ainsi le mérite de poser le vrai problème:

L'armorial de France descend dans l'arène, fait des pirouettes, crie: "Miousic ! miousic ! » exprès pour le divertir (...) Ce dont il se plaint justement, c'est qu'on lui fasse la mariée trop belle. On lui aurait servi le saut des tonneaux avec M. Durand ou

M. Lambert, qu'il se serait contenté de hocher la tête ou de hausser les épaules; hélas!

la bourgeoisie n'est pas en cause, mais bien l'aristocratie, la noblesse ( ...); passe

encore qu'un roturier s'enfarine, mais un prince du sang !

Au cœur de la tourmente, le comte Hubert de la Rochefoucauld: incontestablement le mieux né de la troupe, puisque issu d'une famille reconnue comme appartenant aux plus anciennes et aux plus glorieuses de France, ${ }^{29}$ à ce titre, c'est par lui que le scandale arrive. ${ }^{30} \mathrm{Ce}$ compagnon de la première heure de Molier, avec lequel il a conçu le principe du cirque mondain, est un gymnaste émérite (Vaux 1888b, pp. 109-115). Il choisit donc de s'investir dans d'audacieux numéros de barres fixes et de trapèze volant et s'aventure parfois à jouer les joyeux drilles, grimé en clown et réclamant la
Modern \& Contemporary France 505

musique avec l'accent anglais, ce qui lui attire les quolibets grinçants du Voltaire, le 8 janvier 1885: "À lui la palme, à lui le pompon, à lui le toupet frisé ! ”.

Il est intéresant des critiques de la presse se concentrent sur les 11 est interessant de constater que les cricent exécutés à pied et épanent les numéros dedresse, de force et de noble semble pouvoir se produire dans un cirque numéros équestres. ${ }^{31}$ A cheval, un noble semble pouvoir se et le voilà dépourvu de son sans trop déroger à sa condition. Qu'il mette pied à terre, et le voila depourvu de son identifiant culturel le plus emblématique. En délaissant sa plus noble conquête, il renonce à lui emprunter la mâle autorité que ce fidèle compagnon lui assure depuis des temps immémoriaux pour lui substituer la sienne, en propre, déployée avec ex l'exercice de sa puissance physique quand il se fait gymnaste ou hercule. Au-delà de la traditionnelle recherche de la prouesse, détentrice d'un prestige hercule. Au-dela de lat une rupture culturelle en faisant fructifier son capital viril de bon aloi, il introduit un ferce musculaire. Cette intrinseque publique du corps masculin, qu'il soit admiré ou vilipendé, fascine. irruption publique du corps masculin, qu'il soit admire ou vilipendé, fascine. L'anatomie de La Rochefoucauld est ainsi fort appréciée: Robert Milton relève qu'il est "remarquablement taillé en jeune athlète et qu'il a des muscles d'acier ", tandis que Jules Claretie s'abandonne à la séduction dégagée par ce jeune homme "blond élégant, bien musclé, joli garçon " (Le Figaro 1880 et Le Temps 2-3 janvier 1885). Le maillot d'athlète ou d'hercule, qui dénude autant que le maillot de bain mais qui se donne à voir hors de l'espace consacré de la plage, attire les regards par son incongruité donne a voir hors de lespe counes gens du monde. Si certains se contentent de souligner orsqu'il est porté par des jeunes gens du món sou maillot brun ", d'autre un corps " moulé » ou " admirablement découple dans son maillot brun ", dautres plus précis, remarquent la «culotte avantageuse " (Le Voltaire 1886; Le Temps 2-3 janvier 1885 et Le Figaro 1886). Faut-il croire ces messieurs, si réceptifs aux appât charnels de leurs semblables, quand ils soutiennent que ce sont les femmes qui 'émeuvent d'une virilité si prometteuse? $?^{32}$ Dans une période où les hommes s'affolen de ne pas "prévoir jusqu'où s'émancipera la virilisation de la femme " (Mendes 1885) ${ }^{33}$ la préface donnée par Alexandre Dumas aux Hommes de sport du baron de 1885), la preface donne polier en observant que Vaux, prend tout son sens quand il álégance, cette beauté de l'homme ont le plus gran " cette vigueur, cette adresse, cette elegance, cette beante dans la question ". Plus brutal,

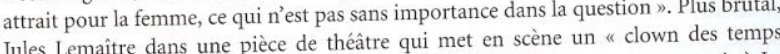
modernes, [un] Léotard des classes dirigeantes " qui ressemble trait pour trait à La modernes, [un] Léotard des classes dirigeantero apole du corps biologisé et de l'énergie Rochefoucau
sexuelle:

C'est une chose délicieuse que d'être un bel animal, aux mouvements souples et for et d'en avoir conscience (...) Si quelque jour une femme digne d'être aimée se rencontre sur son chemin [du gymnaste, soyez tranquile, ne s'en plaindra pas no d'avoir
plus. $^{34}$

à la débauche d'efforts physiques consentis sur la piste, les réaction Face à la des nombreux semble-t-il, légitiment les numéros de force et d'adresse en les rattachant aux saines habitudes 
506 G. Houbre

athlétiques de la Grèce ancienne et en rappelant la prédilection pluriséculaire de l'aristocratie pour les exercices du corps.$^{35}$ Ils se félicitent de cette belle vigueur qui doit avoir raison des «petits crevés » fin de race (Le Temps 2-3 janvier 1885 et Claretie $1883)^{36}$ qui doit triompher de cette dégénérescence sans cesse rebattue depuis plusieurs décennies (Le Temps 11 janvier 1885 et Le Figaro 1884). Beaucoup de notables qui tentent d'exorciser l'humiliation de la défaite de 1870 par l'expression notables, qui gle la gymnastique comme d'un nationalisme plus ou des désastres " (Vigarello 2000, p. 108); aussi n'est-il pas réaction possible au " choc des désastres " (Vigarello 2000, p. 108); aussi n'est-il pas étonnant de voir Gérard de Cherville se réjouir, dans Le Temps da 11 janvier 1885 , de ces "passe-temps virils qui font ces hommes solides dont la patrie a besoin ". Le bonapartiste Paul de Léoni, dans un registre certes vio

exprime la même idée dans L'Autorité daté du 11 juin 1886:

Quand la République aura chu, nous retrouverons ces gymastes et ces acrobates pleins de vigueur et de bravoure. Il ne seront pas amollis dans le plaisir qui dévirilis teins de ve la génération de crétins athées,

de goitreux laïques que Marianne s'évertue à nous préparer.

Cette tendance à la biologisation des corps s'inscrit dans une réflexion générale menée

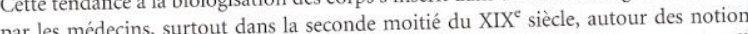
par lendemain de la Première guerre mondiale, elle de degenerscence et de race. Au les déories eugénistes d’un médecin qui trouvera un aboutissement saisissant dans les theories eugénistes d un véritable haras veut améliorer et contrôler la qualité des accouplements en creant un veritable haras humain. Poussant jusqu’à son terme l'identification de la noblesse au cheval, it imagine, en lieu et place de « l'almanach de Gotha, qui ne fait que rappeler les mérites de l'ascendance ", l'instauration d'un « man-book, analogue au stud-book des coursiers et qui comprendra, non seulement le pedigree de chaque membre de l'élite, mais aussi ses performances physiques et intellectuelles " (Binet-Sanglé 1918, p. 239).

Les contempteurs, quant à eux, fustigent le " désir de paraitre qu'Agrippa d'Aubigné Les contempteurs, quant à eux, tus temps ". ${ }^{39}$ Ils s'inquiètent de voir les jeunes gen reprochait deja anx noblains» (Le Temps 13 juin 1886 et $I e$

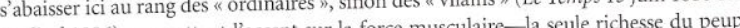
Radical 1886), en mettant l'accent sur la force musculaire-la seule richesse du peuple prolétarien — au détriment de l'intellect et des responsabilités politiques. Anatole France, désolé, s'exclame: «Vraiment ils font pitié. (...) Ils ne s[ont] que des bras; on voudrait qu'ils fussent des têtes " (Le Temps 13 juin 1886), et Henry Fouquier, a bea jer

les mânes de l'ancêtre du gymnaste pour l'heure le plus célèbre de Paris:

Le duc de La Rochefoucauld, qui préférait la gymnastique de l'esprit aux exercices de Le duc de La Roch l'humeur inquiète de la démocratie à venir par ces paroles trapezze, justifait Hhan soutenir \%. (Le Figaro 28 mai 1886) (10 $^{40}$

Ainsi l'initiative privée de Molier, qui n'avait d'autre ambition que de se divertir entre gens du monde, a pris à son grand dam une tournure éminemment politique:

Je ne puis m'empêcher de manifester ma surprise d'avoir été posé par les uns en

Jégénérateur de l'humanité, par les autres, en démoralisateur d'une race. Il ne m'est

508 G. Houbre

maison d'habitation paraissait presque modeste, avec ses deux étages et ses cinq pièces-cuisine, « Calepins des propriétés bâties de $1876 \%$, rue Bénouville (AD/S: DIP4/107).

[10] C'est l'évaluation donnée par Claretie 1883, p. 230.

[11] Sur la pratique du sport par les élites, voir Saint-Martin 1989, pp. 22-32

[12] Léotard créa la voltige sur trapèze volant au cirque Napoléon en 1859 et rencontra immédiatement un vif succès auprès du public parisien.

[13] On peut avoir une idée assez précise de ces numéros en consultant le fonds " Cirque Molier * qui contient de nombreux programmes et comptes-rendus de la presse, ainsi que l'ouvrage de Molier 1905a, qui s'arrête cependant en 1904.

[14] En 1880, les écuyers étaient les comtes de Beauregard, de Pully, de Maulde, de Montherot, baron de Bizy, le capitaine Paret et le lieutenant Bourgeois.

[15] Henri Martell, Maurice Acloque, Jules Ravaut, Guillaume Hugues, ainsi que MM. Durand Vavasseur, Wagner: on les trouve presque tous cités (nommément ou par leur famille), comm leurs compagnons aristocrates, dans Tout-Paris, annuaire de la société parisienne, 1886

[16] « J'avais bien quelques légitimes de mes amis, enragées chasseresses (...) qui me promettaien une foule de merveilles: sauter des barrières ou faire plusieurs tours de manège au galop, les mains en l'air, le buste renverse en arriere et les cheveux au vent $m$ : on perçoit ici sans peine l'ironie dévalorisante de Molier quand il est question des talents des femmes, Molier 1905a, pp. 7, 8; le baron de Vaux s'y montre davantage réceptif dans son ouvrage sur Les Femmes de sport 1885

[17] Le carton d'invitation pour la « soirée » stipule ainsi: « On fera un peu d'équitation, d'acrobatie, etc., et après on mangera des tripes à la mode de Bénouville » ("Cirque Molier $n$ ).

[18] Article de La Liberté, cité par Molier 1905, p. 10. «Le dessus du panier du monde du sport, du monde des cercles et des salles d'armes n pour Paris-Journal, 1881, qui relève également parmi les trois à quatre cents invités une cinquantaine de " dames artistes ".

[19] Le rapprochement des femmes du monde et de celles de l'autre lors de certaines occasions,

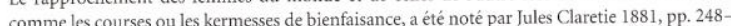
249: “On illumine à la fois le fronton des cercles de high life et les guirlandes du bal Mabille et il y a dans ce rapprochement plus de philosophie qu'on ne croit ".

20] Ragots que Molier appelle pudiquement des " petits nuages », 1905, p. 19

21] * Tout Paris voulut être de la petite fête qui, au lieu de demeurer intime, prit les proportio d'une solennité artistique, à laquelle assista l'élite du monde élégant », Vaux 1888a, p. 110.

22] Voir par exemple Lemaitre 1889; Guinon 1901; Bonnières 1885 .

23] La droite compte 201 députés élus contre 383 pour la gauche en 1885, quand elle en avait 90 contre 461 en 1881.

24] Louis Veuillot, directeur emblématique du journal, est mort en 1883 .

25] Les journaux cités sont Le Figaro, Le Matin et L'Indépendance belge.

[26] Voir par exemple Le Voltaire (gauche gambettiste) 1885 et 1886; L'́cho de Paris 1885; Le Rappel (gauche radicale) 1885: Le Temps 1885 et 21 mai 1886; Le Radical 1886: L'Autorité (bonapartiste (gauche radicale) 1885 , Le Tonss 1886 ainsi que le dossier de presse rassemblé par Molier 1905, pp. $185-192$.

127] Notamment Anatole France (Le Temps 13 juin 1886), et Henry Fouquier qui, dans un article à la sévérité remarquée, se désolidarise de la ligne du Figaro, jusque-là acquis à Molier (28 mai 1886); Émile Blavet, sous le pseudonyme de Parisis, lui répond d'ailleurs dans le même journal ( 2 juin 1886).

28] Le Nenait d'être fondé par Joseph Oller, rue Saint-Honoré. Le Gaulois (1886), journal royaliste du grand monde par ailleurs soutenu financièrement par la duchesse d'Uzès, donne un compte rendu dithyrambique de la fête avec le détail de la location des loges. Notons par exemple la presence des duchesses de Bisaccia, de la Roche-Guyon, de La Tremollo G dAlbuftra, jamais venu à l'idée, en faisant du cirque, de créer une classe dirigeante assez solide livrant à des sports tels que l'équitation, la gymnastique, la force et lescrime, voir méme lacrobatie et le dressage destinées politiques. (Molier 1905, p. 192)

Si la droite modérée se contente de sermonner la conduite de cette jeunesse bien née Si cffense l'ordre social, que ces héritiers de tant de gloire et d'honneur étaient qui " spécialement designes pour dinfluence républicaine qui, dans les annees 1880 , tente p̀es itres, ${ }^{42}$ utilise avec opportunité la symbolique de la noblesse en s'en prenant a ses titres, 'utilise avec opport le noblesse polémique autour du cirque Molier: stigmatisant "l'etape derniere de la noblesse française (...) descendue aux déhanchements du cirque ", elle l'érige en preuve de son discrédit et de son incapacité à conserver un rôle de

dirigeantes (Le Radical 1886 et Le Rappel 1885).

Quand éclate la Grande guerre, les esprits se sont apaises deja depuis longtemps. Le cirque Molier reprend ses activités et retrouve la faveur des chroniques mondaines, et uniquement mondaines: l'aristocratie, en déclin, n'est plus assez puissante pour mériter la controverse et le cheval, concurrencé à la ville par la voiture, a rabattu de sa mériter la controverse et le Molier, en 1933, la presse, nostalgique, célèbre à côté de superbe. A la mort de noble troupe l'expérience unique d'un cirque où "ce fut noble de la Belle Époque où le cheval, d'amateurs ", le représentant d'un autre âge, celui de la Belle Epoque oú le cheval, associé aux élites et au (Le Temps 4 août 1933).

Notes

[1] Voir Bourget 1886, p. VI et Weber 1986 . Ǵ.

[2] Le 4 mai 1844 exactement, selon son acte de decès, conserve al a mainè l'annonce de sa mort, Molier a été assez coquet pour dissimuler sa date de naissance et, apres lennonce de sa moiques $1^{\text {er }}$ août 1933 , les spéculations all

dans le fonds "Cirque Molier ").

[3] Molier est rentier, et son acte de décés,

qualité de directeur d'un cirque privé. [4] Voirl'

1993.

[5] Vaux 1893, pp. 333-339 et 1888a, pp. 109-113. Paul 933 . Blanche Allarty a présenté pour la cirque (1932), in Dossier Molier et Paris Midi, J ate sur une chamelle en 1900, remportant un première fois son numéro d' ' equitation

triomphe auprès du public (LIhustre Panisin

[6] Paul Haynon, dictionnaire des artistes de cirque (

[7] L'Indépendance belge citee par LUnives 8] Lacte de décès de Molier mentionne leepouse sous te nom de 1978, pp. 404-412.

Allarty. Sur les ecuyeres de cirque, voir 9] $247 \mathrm{~m}^{2}$ sur 541,2 exactement; la propriété acquise par Molier en juin 187 remise pour six voitures), la

Thild, les princes Henri d'Orléans et de Saxe-Cobourgmarquise de Galiffet, la baronne de Rothschild, les princs ucs d'Uzèset de la Trémoille, les comtes Gotha; parmi les commissaires, officiaient notammentes et de Chabrol. Enfin, le duc de Crussol, de Montgomery et de Maillé, les vicomtes de Flavigny ea de la troupe de Molier. La soirée aurai deuxième fils de la duchesse d'Uzès, rejoignit pour loccasion la troupe de L'Artet la mode (1886) et rapportel la somme rondelette

[29] " Tant dans l'Église que dans l'Épée ", précise le Dictionnaire de la noblesse de La Chenaye Desbois et Badier, 1772, qui fait remonter la famille au sieur Foucauld I, seigneur de La Roch en 1019 .

[30] Voir Le Rappel (1885) qui évoque « un descendant des croisés » et « quatre messieurs dont les aïeux étaient peut-être à Fontenoy ", Le Radical (1886), qui imagine " que l'ombre de Godefro de Bouillon a tressailli dans sa tombe lorsqu'elle a su que les petits-fils de ses compagnon de Bouilton a tressill dans 'exercices ", mais aussi Le Figaro (28 mai 1886), Le Voltaire (1886), Le Temps (13 juin 1886), ainsi que Dalsème (1888, p. 115).

1 Le lemps Molier, encore une fois, donnait priorité au cheval.

32] «L'orgueil d'étaler sous les regards curieux des femmes, des costumes pailletes d' or qui moulent les torses puissants (...) la tentation exquise du péril bravé au milieu des battements de ces petites mains fines et douces qu'on espère ensuite couvrir

Voir aussi Le Temps 1885
Voir aussi Maugue 1987.

[33] Voir aussi Maugue 1987

54] Ft la dame à qui ce discours s' dresse de s'écrier «Venez à mon secours! On me souille, on me viole et je me meurs de honte ! », 1889, pp. 10-11.

35] Entre autres Houssaye, préface à Vaux 1885, p. II; Le Figaro 7 février 1884 et Le Temps 11 janvier 1885 .

36] Sur ce theme, voir Borie 1981, p. 161.

[37] Voir aussi L'Écho de Paris 1885 .

[38] Voir la mise au point de Carol 1995, chap. 4 « La dégénérescence ".

[39] Henry Fouquier, dans Le Figaro (28 mai 1886), en pointant ce "volontariat du cabotinisme », se

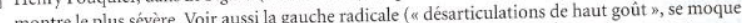
列 Le Radical 1886), (" histrionisme », stigmatise ainsi Édouard Drumont 1886).

la droite extreme (" histrionisme n, stigmatse aimitios les plus violentes. Le correspondant

40] Hubert de la Rochefoucauld a cé la cose des civequ 1885), le chahute en rapportant qu'il ne parisien de Lindépendance belge (cité par L Uniss sur les reins l'intéresse plus que celui de la a s'occupe pas de politique, et que le rétablissement sur les reins linteresse plus que celui de la monarchie ". C'est cette même image, caricaturée, que véhicule la pièce de Guinon (1901), qui le peint de façon peu flatteuse sous les traits du Prince Enguerrand de Barfleur; Guinon, dans cette pièce violemment antisémite, fait du cirque Molier d'une part, de lalliance avec la haute bourgeoisi fit française.

[41] Voir aussi Le Figaro 28 mai 1886

42] Rappelons qu'en 1858, Napoléon III, qui tentait de rallier la noblesse à son régime, fit abroger les décrets de naissance, de classe ou de caste; par l'article 259 du Code Pénal, il voulut lui reconnaitre la légitime possession de ses titres et les protégea contre toute usurpation. C'est pour abroger cet article que le député Beauquier déposa, en vain, un projet de loi en 1882, puis en 1885. Voir article que le depro 270, 279. Anatole France fait également état d'une demande de suppression des titres nobiliaires par le conseil municipal de Paris (Le Temps 13 juin 1886).

[43] Voir les articles nécrologiques consacrés à Molier dans le fonds "Cirque Molier " de la bibliothèque de l'Arsenal, Ro 16749-16757. 


\section{References}

Ami du peuple du soir (3 août 1933)

Ami du peuple du soir (11 août 1933).

André, E. (1908) LEducation physique et sportive des jeunes filles, Flammarion, Paris. Anonyme (1933) 'Un homme de cheval: Ernest Molier', Action francaise, 11 aout L'Art et la mode (12 juin 1886).

Binet-Sanglé, C. (1918) Le Haras humain, Albin Michel, Paris.

Bonnefon, J. de (1910) La Noblesse de France et les anoblis de la République, Michaud, Paris. Bonnières, R. de (1885) Les Monach: roman parisien, Ollendorf, Paris.

Borie, J. (1981) Mythologies de l'hérédité au XIXe siècle Galilée, Paris.

Bourget, P. (1886) Nouveaux essais de psychologie contemporaine, Lemerre, Paris.

Brunay (1886b) 'Sauts de carpe', Le Voltaire, 29 mai.

Carol, A. (1995) Histoire de l'eugénisme en France. Les médecins et la procréation XIX'-XX' sièch Seuil, Paris.

Cherville, G. de (1885) La vie à la campagne, Le Temps, 11 janvier.

Cirque Molier: fonds de la bibliothèque de l'Arsenal, Ro 16749-16757.

Le Clairon (8 février 1884).

Claretie, J. (1883) 'Chronique du 11 mai 1883', in La Vie à Paris, Havard, Paris. in, A. (1995) 'Du loisir cultivé à la classe de loisir', L'Avènement des loisirs 1850-1960, Aubier

Dalsème, A. (1888) Le Cirque à pied et à cheval, Librairie illustrée, Paris

Dossier Molier Archives Départementales/Seine, D28Z/4: Histoire du cirque.

Drumont, É. (1886) La France juive, Flammarion, Paris.

Ferry, L. (1900) 'Au Cirque Molier', L'Illustré parisien, 14 avril.

Le Figaro (6 mai 1883).

Le Figaro ( 7 février 1884).

Le Figaro (3 août 1933).

Fouquier, H. (1886) 'Les Gentilshommes trapézistes', Le Figaro, 28 mai.

Le Gaulois ( 9 février 1884).

Le Gaulois (8 juin 1886).

Guinon, A. (1901) Décadence, comédie en 4 actes, Librairie théâtrale, Paris.

Houbre, G. (1996), 'Les Espaces parisiens de la civilité amoureuse dans la première moitié du XIX siècle', Les Espaces de la civilité, éditions interuniversitaires, Mont-de-Marsan.

Houbre, G. (1998) 'LẤge des amazones', L'Éducation des filles au temps de George Sand, Artois Presses Université, Arras.

Houbre, G. (2003) Histoire de la grandeur et de la décadence de Marie Isabelle, modiste, dresseuse de chevaux, femme d'affaires, etc., Perrin, Paris.

Houssaye, A. (1885) Préface au livre du baron de Vaux, Les Femmes de sport, Flammarion, Paris. Houssaye, A. (1885) Préface au lice
L'tllustré théâtral (15 mai 1897).

Klejman, L. \& Rochefort, F. (1989) L'Égalité en marche. Le féminisme sous la Troisième République,

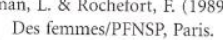

La Chenaye-Desbois de et Badier (1772) Dictionnaire de la noblesse, Schlésinger, Paris. Laerte (1886) 'Hommes et choses. Dans le grand monde', Le Radical, 25 mai. Lemaitre, J. (1889) Révoltée, pièce en 4 actes représentée pour la première fois le 9 avril 1889 sur le théâtre de l'Odéon, Calmann Lévy, Paris.

Léoni, P. de (1886) 'Chroniqué, LAutorité, 11 juin.

Le Roux, H. (1889) Les Jeux du Cirque et la vie foraine, Plon, Paris.

Maizeroy, R. (1885) 'Notes d'un Parisien', LEcho de Paris, 8 janvie.

Maugue, A. (1987) L'Identité masculine en crise au tournant du siècle, Rivages, Paris.
Maver, A. (1983, édn originale 1981) La Persistance de l'Ancien Régime. L'Europe de 1848 a la Grande

Guerre, Flammarion, Paris.
Mendès, C. (1885) Préface au livre du baron de Vaux, Les Femmes de sport, Flammarion, Paris. Milton, R. (1880) 'Le cirque Molier', Le Figaro, 6 juillet.

Molier, E. (1905) Ciraue Molier 1880-1904, Dupont, Paris.

Molier, E. (1911) L'Équitation et le cheval, Lafitte, Paris.

Montargis, E (1885) 'La colère de L'Univers', Le Rappel, 10 janvier.

Non $\mathrm{R}$. (1993) Masculinity and male codes of honor in modern France, University of California Press, Berkeley.

Parisis (1886) 'La Vie parisienne. La fete du Nouveau Cirque', Le Figaro, 2 juin.

Paris-Tournal (21 juin 1881).

Paris Midi ( 3 août 1933) article de Jean Barois.

Pour bien faire du sport (1912), Lafitte, Paris.

Pon

(I) noblesse et les 'sports' nobles', Actes de la recherche en sciences sociales, no. 80 , novembre.

Serizie, Le (1885a) 'Les clowns du grand monde', Le Voltaire, 8 janvier.

Serizier, L. (1885a) Les clowns du grand

Le Temps (21 mai 1886).

Le Temps (13 juin 1886) article d'Anatole France.

Le Temps (3 août 1933).

Le emps (4 aout 193).

Tout-Paris, annuaire de la société parisienne (1886), La Fare, Paris.

Vaux, Baron de (1885) Les Femmes de sport, Flammarion, Paris.

Vaux, Baron de (1888a) Les Hows

Vaux, Baron de (1888b) Les Hommes de sport, Flammarion, P

Vaux, Baron de (1893) Eccuyers et écuyeres, Rothschild, Paris.

Vigarello, G. (2000) Passion sport. Histoire d'une culture, Textuel, Paris. 